\title{
Pyomyositis - A Short Review
}

\section{Lasitha B Samarakoon}

Resident in General Surgery, Singapore General Hospital, Singapore

*Corresponding author: Lasitha B Samarakoon, Resident in General Surgery, Singapore General Hospital, Singapore, Tel: +6586610121; E-mail: lasithamfc@gmail.com

\section{Received date: December 15, 2015; Accepted date: February 01, 2016; Published date: February 05, 2016}

Copyright: ( 2016 Samarakoon LB. This is an open-access article distributed under the terms of the Creative Commons Attribution License, which permits unrestricted use, distribution and reproduction in any medium, provided the original author and source are credited.

\section{Introduction}

Tropical pyomyositis (TPM) is a disease characterized by suppuration occurring within skeletal muscle, resulting in single or multiple abscesses within large muscle groups Although initially reported exclusively from the tropics, worldwide incidence is increasing as more and more cases are diagnosed with increasing awareness about the condition. A short review with review of literature is presented on this paper, in order to create awareness about this lethal albeit potentially curable condition.

Looking back into the history of Tropical pyomyositis, earliest description dates back to 1885 , when Scriba allured to it in one of his papers [1]. First case in tropics was reported by Levin, as far back as 1971 [2], and since then number of cases reported from outside tropical areas has steadily increased $[3,4]$.

Due to initial recognition of this condition from the tropics, various terminologies such as tropical pyomyositis, myositis tropicans were used in literature to describe the condition. Since increasing reporting from the temperate regions terminology such as Non tropical pyomyositis as well as infectious myositis are increasingly in use nowadays. It is extremely important to use correct terminology as TPM does not encompass intramuscular abscesses, abscesses extending to muscle from adjacent structures such as bone or secondary skeletal muscle abscesses secondary to septicaemia.

TPM contributes to nearly $5 \%$ of hospital admissions in tropical countries such as Uganda [5]. In temperate regions TPM is increasingly recognized in association with immunodeficiency states such as HIV, Diabetes as well as Leukemia [6]. Infact one study quoted incidence of TPM to be high as $31 \%$ in HIV infected patients, with or without AIDS [6].Minor trauma affecting the skeletal muscle groups also seem to predispose patients towards TPM $[7,8]$.

Microbiologically Staphylococcus aureus seems to be the most common microorganism associated with TPM, 90\% in tropical countries and $75 \%$ of temperate cases [3]. Next commonest is group A streptococcus, accounting for $5 \%$ of cases [3]. Rarer cases include Salmonella, mycobacterium and anaerobes as the causative organism $[9,10]$. In tropics nearly $30 \%$ of pus cultures are negative and $90 \%$ of blood cultures are reported as sterile [11].

TPM is an intriguing disease in the sense that skeletal muscle is intrinsically resistant to colonization by blood borne bacteria [11] Immunodeficiency is an important predisposing factor for TPM [12], with CD4 $<150 /$ micro liter an important predisposing factor. Staphylococcal carriage has been shown to be more common among HIV infected patients than otherwise [13], and may partly explain this difference.

Pathologically, early tropical pyomyositis, muscle fibers show edema and separation of fibers, progressing to myositis and progressive lysis.
Lymphoplasmacytic infiltrate is often noted. As a sequale muscle fibers may heal without abscess formation or degenerate, progressing to suppuration [14].

TPM affects adults most commonly in 10 to 40 year age group with a slight preponderance of males over females. Larger muscle groups such as glutei, pectoralis as well as smaller muscle groups such as biceps may be affected [15]. Although the normally, single muscle groups are affected, in about one third, multiple groups may be affected $[16,17]$.

Clinically TPM is characterized by three stages, although atypical presentations are not uncommon. Initial Flu like illness characterizes invasive stage, without any definite abscess formation. This stage may mimic cellulites, thrombophlebitis or even fibromyalgia. Next is the suppurative stage, although classic features of abscess formation such as fluctuation may not be obvious due to the overlying tense muscle groups. Untreated disease may progress to septicaemia, metastatic abscess formation and multi organ failure leading to shock.

Atypical presentation of TPM with spinal cord compression [18] or compartment syndrome [13] has been documented. TPM affecting the neck muscles may mimic cerbrobrachial neuralgia [19].

Majority of morbidity associated with TPM is due to delay in diagnosis, leading to systemic sepsis. Although aspiration and culture of pus from abscesses and isolating Staphylococci from muscle biopsies is the gold standard of diagnosis this is not always possible in early stages of disease [20]. Blood culture may be positive in up to $10 \%$ in tropics and $30 \%$ in temperate regions due to if the clinical suspicion is high noninvasive imaging modalities can be used early as an adjunct to diagnosis. Although ultrasound may demonstrate hypo echoic masses within muscles, Magnetic Resonance Imaging is the gold standard for early diagnosis [21], distinctly better than Computed tomography in differentiating between abscess and muscle swelling [22]. Even though one would expect the muscle enzymes such as creatinine phosphokinase, Aldolase, amino transferase and lactate dehydrogenase to be greatly elevated given the context of muscle necrosis, they may be normal or show slight derangement only [23].

Once diagnosis is made emphasis is placed on drainage of pus and appropriate parenteral antibiotics. If the strain is sensitive Penicillin is the drug of choice. In case of penicillin allergy, ceflazolin may be substituted. In case of methicillin resistant staphylococci, Vancomycin or Teicoplanin may be used to good effect. Linezolid is an alternative for Vancomycin intermediate enterococci. Usually antibiotic treatment is continued for 4 to 6 weeks parenterally. There is some evidence for eradication of nasal carriage of staphylococci in patients with TPM [24].

Despite advances in diagnosis and treatment mortality of $2 \%$ is still quoted for TPM. Prognosis becomes significantly worse with any delay 
in diagnosis [25], hence the importance of prompt diagnosis and institution of appropriate treatment.

\section{References}

1. Scriba J, Zur B (1885) Aetiologie der myositis acuta. Deutsche Zeit Chir 22: 497-502.

2. Levin MJ, Gardner P, Waldvogel FA (1971) An unusual infection due to staphylococcus aureus. N Engl J Med 284: 196-198.

3. Christin L, Sarosi GA (1992) Pyomyositis in North America: case reports and review. Clin Infect Dis 15: 668-677.

4. Gibson RK, Rosenthal SJ, Lukert BP (1984) Pyomyositis. Increasing recognition in temperate climates. Am J Med 77: 768-772.

5. Smith PG, Pike MC, Taylor E, Taylor JF (1978) The epidemiology of tropical myositis in the Mengo Districts of Uganda. Trans R Soc Trop Med Hyg 72: 46-53.

6. Hossain A, Reis ED, Soundararajan K, Kerstein MD, Hollier LH (2000) Nontropical pyomyositis: analysis of eight patients in an urban center. Am Surg 66: 1064-1066

7. Jayoussi R, Bialik V, Eyal A, Shehadeh N, Etzioni A (1995) Pyomyositis caused by vigorous exercise in a boy. Acta Paediatr 84: 226-227.

8. Diamandakis V, Grose C (1994) Bad consequences of bicycle accidents. Pyomyositis. Pediatr Infect Dis J 13: 422-425.

9. Sarubbi FA, Gafford GD, Bishop DR (1989) Gram-negative bacterial pyomyositis: unique case and review. Rev Infect Dis 11: 789-792.

10. Mootsikapun P, Mahakkanukrauh A, Suwannaroj S, Nanagara R (2003) Tuberculous pyomyositis. J Med Assoc Thai 86: 477-481.

11. Brown JD, Wheeler B (1984) Pyomyositis. Report of 18 cases in Hawaii. Arch Intern Med 144: 1749-1751.

12. Rodgers WB, Yodlowski ML, Mintzer CM (1993) Pyomyositis in patients who have the human immunodeficiency virus. Case report and review of the literature. J Bone Joint Surg Am 75: 588-592.
13. Aynaci O, Onder C, Kalaycioglu A (2003) Anterior tibial compartment syndrome due to the pyomyositis in a patient with rheumatoid arthritis. A case report. Joint Bone Spine 70: 77-79.

14. Taylor JF, Templeton AC, Henderson B (1970) Pyomyositis. A clinicopathological study based on 19 autopsy cases, Mulago Hospital 1964-1968. East Afr Med J 47: 493-501.

15. Ashken MH, Cotton RE (1963) Tropical Skeletal Muscle Abscesses (Pyomyositis Tropicans). Br J Surg 50: 846-852.

16. Horn CV, Master S (1968) Pyomyositis tropicans in Uganda. East Afr Med J 45: 463-471.

17. Chacha PB (1970) Muscle abscesses in children. Clin Orthop Relat Res 70: $174-180$.

18. Brik R, Braun J, Bialik V, Zuckerman N, Berant M (1989) Non-tropical pyomyositis in children--with report of severe neurological complications. Acta Paediatr Scand 78: 331-334.

19. Flory P, Brocq O, Euller-Ziegler L, Ziegler G (1993) Pyomyositis: cervical localization. J Rheumatol 20: 1411-1413.

20. Shepherd JJ (1983) Tropical myositis: is it an entity and what is its cause? Lancet 2: 1240-1242.

21. Trusen A, Beissert M, Schultz G, Chittka B, Darge K (2003) Ultrasound and MRI features of pyomyositis in children. Eur Radiol 13: 1050-1055.

22. Gordon BA, Martinez S, Collins AJ (1995) Pyomyositis: characteristics at CT and MR imaging. Radiology 197: 279-286.

23. Gupta B, Khanna SK, Sharma BK (1980) Pyomyositis. J Assoc Physicians India 28: 91-94.

24. Hudson IR (1994) The efficacy of intranasal mupirocin in the prevention of staphylococcal infections: a review of recent experience. J Hosp Infect 27: 81-98.

25. Kerrigan KR, Nelson SJ (1992) Tropical pyomyositis in eastern Ecuador. Trans R Soc Trop Med Hyg 86: 90-91. 